\title{
Neurotrophic Effects of Genipin on Neuro2a Cells
}

\author{
Matsumi Yamazaki*,a and Kenzo Chiba ${ }^{a, b}$ \\ ${ }^{a}$ Department of Biochemistry, Faculty of Pharmaceutical Sciences, and ${ }^{b}$ Organization for Frontier Research in Preventive Pharma- \\ ceutical Sciences, Hokuriku University, Ho-3 Kanagawa-machi, Kanazawa, Ishikawa 920-1181, Japan
}

(Received August 22, 2005; Accepted August 29, 2005; Published online September 1, 2005)

\begin{abstract}
We have found that genipin, a herbal iridoid compound, can induce neurite outgrowth in rat pheochromocytoma PC12h cells. In this study, we examined the neurotrophic effects of genipin in mouse neuroblastoma Neuro2a cells. Genipin significantly induced neurite outgrowth in a concentration-dependent manner in Neuro2a cells cultured in serum-free medium supplemented with transferrin/insulin/progesterone (TIP) without cytotoxicity. Neuro2a cells cultured in serum-containing medium were mitotic, while the proliferation of these cells cultured in serum-free medium supplemented with TIP was suppressed for up to $48 \mathrm{hr}$. Serum and TIP-free medium clearly increased the number of cells stained with trypan blue compared with serum-free medium supplemented with TIP. Damage to the cells was significantly reduced by adding genipin to the cells in serum and TIP-free medium. As a positive control for a neurotrophic action, dibutyryl cAMP, a membrane-permeable cAMP analog, also induced neuritogenesis in cells cultured in serum-free medium supplemented with TIP and enhanced the survival of cells cultured in serum and TIP-free medium. These results indicate that genipin has both neuritogenic activity and a survival effect on serumdeprivation-induced cell death in Neuro2a cells. This suggests that genipin can act as a neurotrophic factor-like compound in neuronal cells.
\end{abstract}

Key words — genipin, neurite outgrowth, survival, Neuro2a cell

\section{INTRODUCTION}

It has been reported that nerve growth factor (NGF) and other neurotrophins play important roles in growth, differentiation, and survival in neuronal cells. ${ }^{1)}$ They, therefore, are expected to have a regenerative action in injured tissues in neurodegenerative diseases such as Alzheimer's disease and Parkinson's disease. However, because of their inability to cross the blood-brain barrier and vulnerability to hydrolytic enzymes, they cannot be used as a medical treatment. Therefore, we have been screening natural lipophilic compounds in plants traditionally used as antiamnestic or anti-inflammatory medications as substitutes for neurotrophins.

Genipin is an iridoid compound, isolated from Gardenia fructus, and the aglucon of geniposide. We have previously reported that genipin markedly induces neurite outgrowth through a nitric oxide (NO)guanosine $3^{\prime}, 5^{\prime}$-cyclic monophosphate (cGMP)-

\footnotetext{
*To whom correspondence should be addressed: Department of Biochemistry, Faculty of Pharmaceutical Sciences, Hokuriku University, Ho-3, Kanagawa-machi, Kanazawa, Ishikawa 920 1181, Japan. Tel.: +81-76-229-1165; Fax: +81-76-229-2781; Email: m-yamazaki@hokuriku-u.ac.jp
}

cGMP-dependent protein kinase (PKG) pathway followed by ERK activation in rat pheochromocytoma PC12h cells. ${ }^{2,3)}$ This signaling pathway was observed in the neurite outgrowth process induced by NGF in PC12h cells. ${ }^{2,3)}$ Moreover, we have suggested that genipin directly binds to and activates neuronal NO synthase (NOS) in vitro. ${ }^{4)}$

In the present study, we have examined the possibility that genipin may be a new neurotrophic factor possessing a neuroprotective effect and may induce neuronal differentiation by investigating the protective effect of genipin on cytotoxicity induced by serum deprivation in mouse neuroblastoma Neruro2a cells.

\section{MATERIALS AND METHODS}

Materials — Genipin, transferrin, and $\mathrm{N}^{6}, \mathrm{O}^{7}$ dibutyryl cAMP (dbcAMP) were purchased from Wako Pure Chemical Industries, Ltd. (Japan). Insulin and progesterone were obtained from Sigma Chemical Co. (U.S.A.). 3-(4, 5-Dimethylthiazol-2yl)-2, 5-diphenyltetrazolium bromide (MTT) was purchased from Dojindo (Japan).

Cell Culture — Neuro2a cells were grown in 
Eagle's minimum essential medium (EMEM) containing $10 \%(\mathrm{v} / \mathrm{v})$ fetal bovine serum in $10 \% \mathrm{CO}_{2} /$ $90 \%$ humidified air at $37^{\circ} \mathrm{C}$. Neuro2a cells were plated onto $35-\mathrm{mm}$ culture dishes for neurite outgrowth assay at a density of $2 \times 10^{3}$ cells $/ \mathrm{cm}^{2}$ or 48 -well culture plates for cell viability assay at a density of $1.5 \times 10^{4}$ cells $/ \mathrm{cm}^{2}$. After $24 \mathrm{hr}$ of culture, the culture medium was replaced with serumfree EMEM with or without $5 \mu \mathrm{g} / \mathrm{ml}$ transferrin/ $5 \mu \mathrm{g} / \mathrm{ml}$ insulin/20 $\mathrm{nM}$ progesterone [transferrin/insulin/progesterone (TIP)] to treat the cells with agents as specified in the results.

Neurite Outgrowth Assay — Neurite outgrowth in Neuro2a cells was evaluated by measuring the length of the longest neurite in individual cells as previously reported. ${ }^{2)}$ The neurite length of 100 cells in 10 random fields from two sister culture dishes was averaged to evaluate the neurite outgrowth for each treatment.

Cell Viability Assay — Cell viability of Neuro2a cells was evaluated by measuring the activity of MTT reduction and staining with trypan blue. For MTT reduction activity, the cells were treated with $0.25 \mathrm{mg} / \mathrm{ml} \mathrm{MTT}$ at $37^{\circ} \mathrm{C}$ for $30 \mathrm{~min}$. The medium was then removed and the reduction product, MTTformazan, was solubilized with dimethylsulfoxide (DMSO). Absorption at $550 \mathrm{~nm}$ of each sample solution was measured as MTT reduction activity of the cells. Data are expressed in terms of the relative activity in relation to each control group. For staining with trypan blue, the cells were exposed to $0.4 \%(\mathrm{w} / \mathrm{v})$ trypan blue at room temperature for $5 \mathrm{~min}$ and then examined with a microscope.

Statistical Analysis — Statistically significant differences between groups were estimated by analysis of variance (ANOVA) followed by Student's $t$ test. A $p<0.05$ was considered to indicate a significant difference.

\section{RESULTS}

\section{Genipin-Induced Neurite Outgrowth}

In previous papers, ${ }^{2,5)}$ we demonstrated that genipin induced neurite outgrowth in PC12h cells. As shown in Fig. 1, genipin $(1-5 \mu \mathrm{g} / \mathrm{ml})$ had significant neuritogenic activity in a concentration-dependent manner in Neuro2a cells cultured in serum-free medium supplemented with TIP as well as PC12h cells.

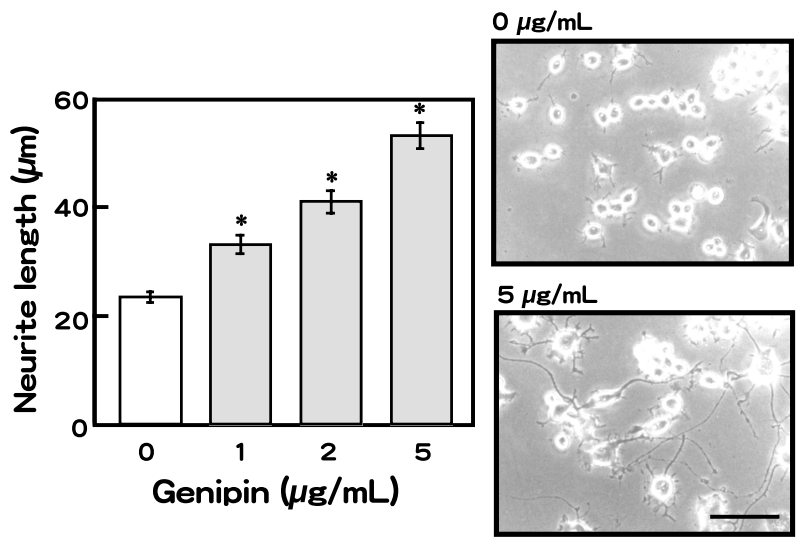

Fig. 1. Genipin-Induced Neurite Outgrowth in Neuro2a Cells Neuro2a cells were cultured for $24 \mathrm{hr}$ and then treated with genipin in serum-free medium with TIP for $48 \mathrm{hr}$. Then, the neurite length was measured as described in MATERIALS AND METHODS and microphotographs were taken (scale bar $=100 \mu \mathrm{m}$ ). Each value is expressed as the mean $\pm \operatorname{SEM}(n=100) .{ }^{*} p<0.01 v s .0 \mu \mathrm{g} / \mathrm{ml}$.

\section{Serum Deprivation-Induced Cytotoxicity}

In general, culture conditions without serum are insufficient for the long-term survival of most neuronal cells. However, it is difficult to use medium with serum for the evaluation of cell survival because of the proliferation of Neuro2a cells. It has been reported that TIP results in lower proliferation in PC12h cells in serum-free medium and no changes in cell properties. $\left.{ }^{6}\right)$ We evaluated cell proliferation by MTT reduction activity of Neuro2a cells cultured in several conditions. The medium with serum induced proliferation in a time-dependent manner, while the serum-free medium with TIP suppressed it at $48 \mathrm{hr}$ of incubation (Fig. 2A). We tested the induction of cytotoxicity in Neuro2a cells cultured in the serum-free medium without TIP and found a significant reduction in MTT activity compared with the medium with TIP (Fig. 2A). To determine whether this reduced activity is due to cytotoxicity, we performed trypan blue staining. After a 48-hr culture in the serum-free medium with or without TIP, the number of stained cells was clearly increased in the medium without TIP (Fig. 2B). Thus, we decided that this serum-free medium without TIP is appropriate for cell cultures with which to examine whether genipin can protect against serum deprivation-induced cytotoxicity.

\section{Protective Effect of Genipin on Cytotoxicity In- duced by Serum-Free Medium without TIP}

We tested the effect of genipin alone on cell survival by measuring MTT reduction activity. Genipin 
A

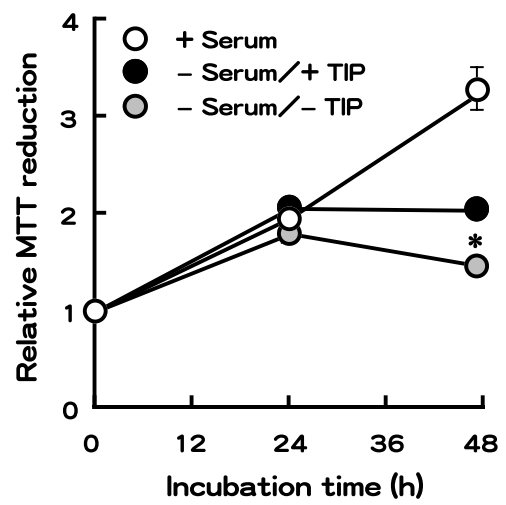

B

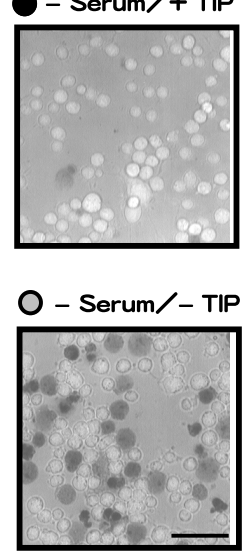

Fig. 2. Cytotoxicity Induced by Serum-Free Medium without TIP in Neuro2a Cells

Neuro2a cells were cultured for $24 \mathrm{hr}$ and then incubated in serum-containing medium (+Serum), serum-free medium with TIP (-Serum/+TIP), or serum-free medium without TIP (-Serum/-TIP) over $48 \mathrm{hr}$. (A) Time course of MTT reduction. Each value is expressed as the mean \pm SEM $(n=3)$. ${ }^{*} p<0.01 v s$. -Serum/+TIP at $48 \mathrm{hr}$. (B) Trypan blue staining at $48 \mathrm{hr}($ scale bar $=100 \mu \mathrm{m})$.

A

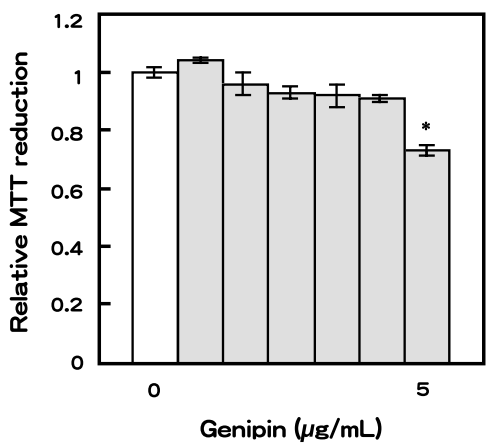

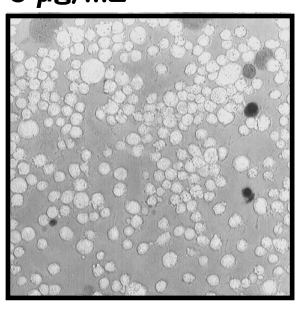

$5 \mu \mathrm{g} / \mathrm{mL}$

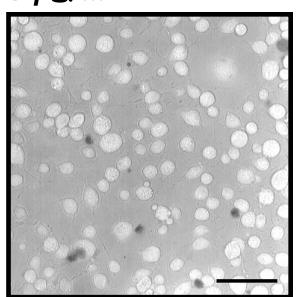

Fig. 3. Effect of Genipin on Cell Survival in Neuro2a Cells

Neuro2a cells were cultured for $24 \mathrm{hr}$ and then treated with genipin in serum-free medium with TIP for $48 \mathrm{hr}$. (A) Concentration-dependency of MTT reduction. Each value is expressed as the mean $\pm \operatorname{SEM}(n=6) .{ }^{*} p<0.01 v s .0 \mu \mathrm{g} / \mathrm{ml}$. (B) Trypan blue staining (scale bar $\left.=100 \mu \mathrm{m}\right)$.

had no effect on MTT reduction activity at concentrations of $0.1-2 \mu \mathrm{g} / \mathrm{ml}$ in Neuro2a cells cultured in serum-free medium with TIP for $48 \mathrm{hr}$ (Fig. 3A). Although $5 \mu \mathrm{g} / \mathrm{ml}$ genipin significantly reduced the activity (Fig. 3A), it did not increase the number of trypan blue-stained cells (Fig. 3B). Thus, we concluded that the reduced activity was not due to cytotoxicity induced by genipin but rather was due to differentiation as supported by Fig. 1. Next, we investigated the effect of genipin on cytotoxicity in Neuro2a cells cultured in serum-free medium without TIP using the MTT reduction assay. As shown in Fig. 4A, genipin significantly protected against cytotoxicity in a concentration-dependent manner. This was consistent with the morphological evaluation as shown in Fig. 4B.

\section{Neurotrophic Effects of cAMP}

It has been reported that cAMP induces neurite outgrowth and neuronal differentiation in Neuro2a cells. ${ }^{7)}$ As a positive control, we investigated the neurotrophic effects, namely neurite outgrowth and cell survival, of cAMP using a membrane-permeable cAMP analog, dbcAMP, in Neuro2a cells. Cells 
A

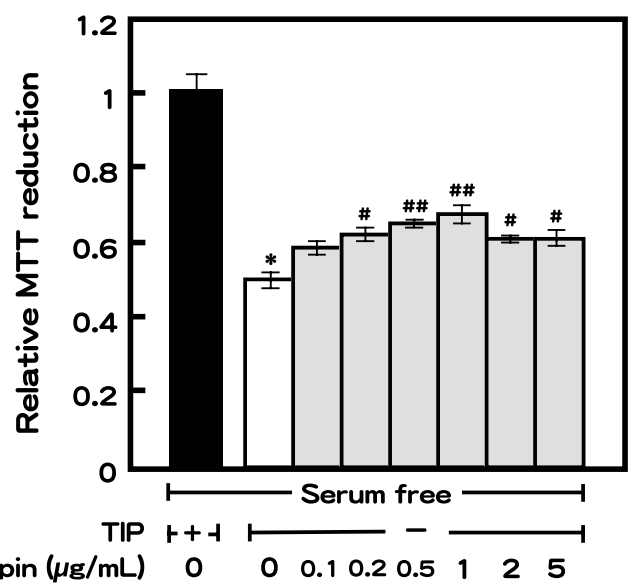

B
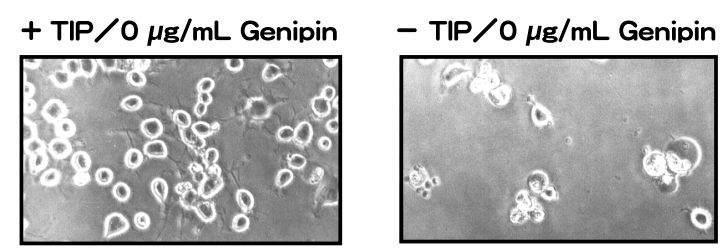

- TIP $/ 1 \mu \mathrm{g} / \mathrm{mL}$ Genipin
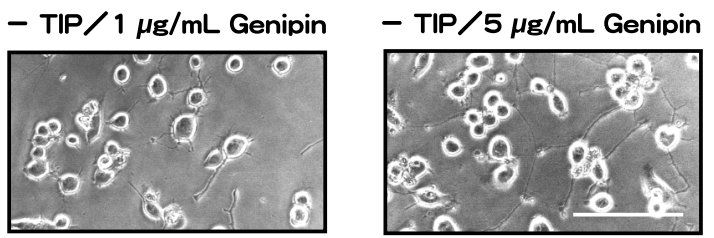

Fig. 4. Protective Effect of Genipin on Cytotoxicity Induced by Serum-Free Medium without TIP in Neuro2a Cells

Neuro2a cells were cultured for $24 \mathrm{hr}$ and then treated with genipin for $48 \mathrm{hr}$ as indicated. (A) MTT reduction activity. Each value is expressed as the mean $\pm \operatorname{SEM}(n=5) .{ }^{*} p<0.01 v s .+\mathrm{TIP} / 0 \mu \mathrm{g} / \mathrm{ml}$ genipin and $\# p<0.05, \# \# p 0.01 v s$. $-\mathrm{TIP} / 0 \mu \mathrm{g} / \mathrm{ml}$ genipin. (B) Morphological changes (scale bar $=100 \mu \mathrm{m})$.

treated with dbcAMP $(1-200 \mu \mathrm{M})$ for $48 \mathrm{hr}$ significantly extended neurites in a concentration-dependent manner (Fig. 5A). Treatment with $500 \mu \mathrm{M}$ or greater concentrations made the cells float (data not shown). The cytotoxicity observed in serum-free medium without TIP for $48 \mathrm{hr}$ was effectively blocked by dbcAMP in a concentration-dependent manner (Fig. 5B).

\section{DISCUSSION}

In the present study, genipin was found to exert not only a neuritogenic action but also a neuroprotective effect on serum deprivation-induced cell death in Neuro2a cells.

We have previously reported that genipin induces
A

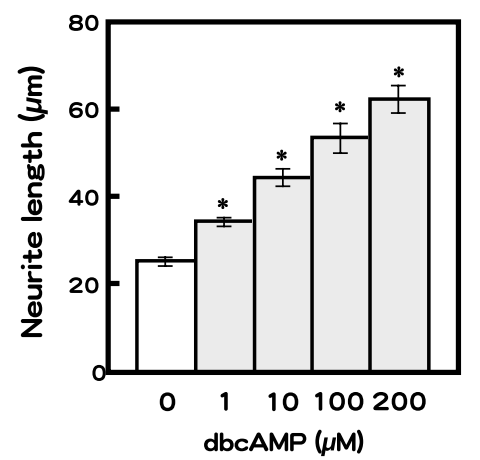

B

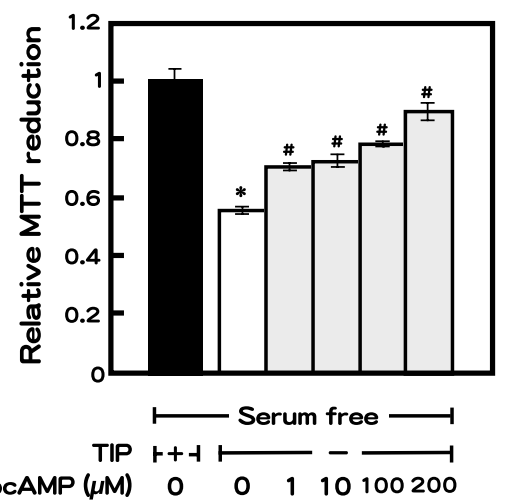

Fig. 5. Neurotrophic Effects of dbcAMP in Neuro2a Cells

Neuro2a cells were cultured for $24 \mathrm{hr}$ and then treated with dbcAMP for $48 \mathrm{hr}$ as indicated. (A) Dibutyryl cAMP-induced neurite outgrowth. Dibutyryl cAMP was added to serum-free medium with TIP. Neurite length was measured as described in MATERIALS AND METHODS. Each value is expressed as the mean $\pm \operatorname{SEM}(n=100) . * p<0.01 v s$. $0 \mu \mathrm{M}$. (B) Protective effect of genipin on cytotoxicity induced by serumfree medium without TIP. The effect was assessed by MTT reduction assay. Each value is expressed as the mean $\pm \operatorname{SEM}(n=8) . * p<0.01 v s$. $+\mathrm{TIP} / 0 \mu \mathrm{M}$ dbcAMP and \# $<0.01 v s$. $-\mathrm{TIP} / 0 \mu \mathrm{M}$ dbcAMP.

neurite outgrowth via the NO-cGMP-PKG pathway followed by ERK activation in PC12h cells ${ }^{2,3)}$ and that neuronal NOS would be a target molecule of genipin in vitro. ${ }^{4)}$ It is well known that $\mathrm{PC} 12$ and its subclonal cell lines such as PC12h show significant neurite outgrowth in response to NGF which binds to its receptor, TrkA. ${ }^{1)}$ To confirm the universality of the neuritogenic action of genipin, we used Neuro2a cells, which are another neuronal cell line. Neuro2a cells are NGF-insensitive and responsive to cAMP, retinoic acid, and gangiosides. ${ }^{8}$ Genipin significantly induced neurite outgrowth in Neuro2a cells as well as PC12h cells. The mechanism of this action in Neuro2a cells is not yet understood. However, it is conceivable that genipin activates neuronal NOS as observed in PC12h cells based on the fact that Neuro2a cells possess neuronal NOS protein (unpublished data). For the present, it seems certain that the genipin-induced neurite outgrowth is not lim- 
ited to only PC12h cells.

The serum-deprived cell is often used as a model of neurodegenerative, pathological cell death. In the present study, we investigated the neuroprotective effect of genipin on neuronal cell death using this model to ascertain whether genipin can act as a neurotrophic factor possessing neuronal differentiation and neuroprotection activities. Genipin significantly prevented serum and TIP deprivation-induced cell death in Neuro2a cells, which was similar to the effect of dbcAMP, an established neurotrophic effector in the cells. It has been reported that serum deprivation induces apoptosis in several cell types..$^{9,10)}$ On the other hand, Yamamoto et al. have reported that genipin suppresses Fas-induced apoptosis in primary cultured mouse hepatocytes. ${ }^{11)}$ They indicated that genipin blocked the activation of caspase 3 and a reduction in mitochondrial membrane potential, which are apoptosis markers. ${ }^{11)}$ Therefore, it is possible that the neuroprotective effect of genipin seen in our experiments was based on the inhibition of these markers.

It has been suggested that NO and cGMP, a downstream component of NO production, play important roles in neuronal survival. For example, several groups have reported that NO protected neuronal cells from serum deprivation-induced cell death through cGMP production. ${ }^{12,13)}$ Taking into consideration the results of our previous reports that genipin directly activates neuronal NOS and produces cGMP in PC12h cells, ${ }^{3,4)} \mathrm{NO}$ and cGMP produced by genipin might act as intermediators to protect Neuro2a cells against serum deprivation.

We have previously found that genipin prevents $\beta$-amyloid peptide-induced neurotoxicity in primary cultured rat hippocampal neurons. ${ }^{14)}$ It has been suggested that one of the common mechanisms between serum deprivation- and $\beta$-amyloid peptide-induced cytotoxicity is the induction of oxidative stress. ${ }^{15,16)}$ Thus, genipin may suppress the cytotoxicity involved in oxidative stress.

In summary, this paper suggests that genipin acts as a new neurotrophic factor-like compound with both neuritogenic and neuroprotective effects in Neuro2a cells. Therefore, genipin has the potential to serve as a lead compound for the treatment of neurodegenerative diseases such as Alzheimer's disease. Further studies are now underway to clarify the mechanisms of these effects of genipin.

Acknowledgements This work was supported by the "Academic Frontier" Project for Private Univer- sities from the Ministry of Education, Culture, Sports, Science and Technology of Japan (20052009).

\section{REFERENCES}

1) Patapoutian, A. and Reichardt, L. F. (2001) Trk receptors: mediators of neurotrophin action. Curr. Opin. Neurobiol., 11, 272-280.

2) Yamazaki, M., Chiba, K., Mohri, T. and Hatanaka, H. (2001) Activation of the mitogen-activated protein kinase cascade through nitric oxide synthesis as a mechanism of neuritogenic effect of genipin in PC12h cells. J. Neurochem., 79, 45-54.

3) Yamazaki, M., Chiba, K., Mohri, T. and Hatanaka, H. (2004) Cyclic GMP-dependent neurite outgrowth by genipin and nerve growth factor in PC12h cells. Eur. J. Pharmacol., 488, 35-43.

4) Ohkubo, T., Yamazaki, M., Yoshida, A., Chiba, K. and Mohri, T. (2004) Detection of genipin/ geniposide-target molecules by a geniposide overlay method using anti-geniposide antibody. J. Health Sci., 50, 193-196.

5) Yamazaki, M., Chiba, K. and Mohri, T. (1996) Neuritogenic effect of natural iridoid compounds on $\mathrm{PC} 12 \mathrm{~h}$ cells and its possible relation to signaling protein kinases. Biol. Pharm. Bull., 19, 791-795.

6) Hatanaka, H. (1983) Nerve growth factor-mediated differentiation of a nerve cell line cultured in a hormone-supplemented serum-free medium. Brain Res. Dev. Brain Res., 6, 243-250.

7) Wong, C. K., Yeung, H. Y., Mak, N. K., DiMattia, G. E., Chan, D. K. and Wagner, G. F. (2002) Effects of dibutyryl cAMP on stanniocalcin and stanniocalcin-related protein mRNA expression in neuroblastoma cells. J. Endocrinol., 173, 199-209.

8) Matta, S. G., Yorke, G. and Roisen., F. J. (1986) Neuritogenic and metabolic effects of individual gangliosides and their interaction with nerve growth factor in cultures of neuroblastoma and pheochromocytoma. Brain Res. Dev. Brain Res., 27, 243-252.

9) Rivera, S., Guillot, S., Agassandian, C., Ben, A. Y. and Khrestchatisky, M. (1998) Serum deprivationinduced apoptosis in cultured hippocampi is prevented by kainate. Neuroreport, 9, 3949-3953.

10) Macleod, M. R., Allsopp, T. E., McLuckie, J. and Kelly, J. S. (2001) Serum withdrawal causes apoptosis in SHSY 5Y cells. Brain Res., 889, 308315.

11) Yamamoto, M., Miura, N., Ohtake, N., Amagaya, S., Ishige, A., Sasaki, H., Komatsu, Y., Fukuda, K., Ito, T. and Terasawa, K. (2000) Genipin, a metabolite derived from the herbal medicine Inchin-ko-to, 
and suppression of Fas-induced lethal liver apoptosis in mice. Gastroenterology, 118, 380-389.

12) Farinelli, S. E., Park, D. S. and Greene, L. A. (1996) Nitric oxide delays the death of trophic factor-deprived PC12 cells and sympathetic neurons by a cGMP-mediated mechanism. J. Neurosci., 16, 23252334.

13) Kim, Y. M., Chung, H. T., Kim, S. S., Han, J. A., Yoo, Y. M., Kim, K. M., Lee, G. H., Yun, H. Y., Green, A., Li, J., Simmons, R. L. and Billiar, T. R. (1999) Nitric oxide protects PC12 cells from serum deprivation-induced apoptosis by cGMP-dependent inhibition of caspase signaling. J. Neurosci., 19,
6740-6747.

14) Yamazaki, M., Sakura, N., Chiba, K. and Mohri, T. (2001) Prevention of the neurotoxicity of the amyloid _ protein by genipin. Biol. Pharm. Bull., 24, 1454-1455.

15) Andoh, T., Lee, S. Y. and Chiueh, C. C. (2000) Preconditioning regulation of bcl-2 and $\mathrm{p} 66^{\text {shc }}$ by human NOS1 enhances tolerance to oxidative stress. FASEB J., 14, 2144-2146.

16) Chong, Z. Z., Li, F. and Maiese, K. (2005) Stress in the brain: novel cellular mechanisms of injury linked to Alzheimer's disease. Brain Res. Brain Res. Rev., 49, 1-21. 